\title{
Trajectory optimization for soft landing of fast-switching electromagnetic valves
}

\author{
T. Glück* W. Kemmetmüller* A. Kugi * \\ * Automation and Control Institute, Vienna University of Technology, \\ Gusshausstrasse 27-29, 1040 Vienna, Austria \\ (e-mail: \{glueck, kemmetmueller,kugi\}@acin.tuwien.ac.at)
}

\begin{abstract}
The design of a feedforward controller that facilitates soft landing and time optimality of a fast-switching electromagnetic valve is presented. In particular, a mathematical model of the considered pneumatic switching valve is developed and parametrized by means of nonlinear parameter identification. Based on this model, the input constrained point-topoint quasi-time-optimal control problem is formulated and the resulting two-point boundary value problem is numerically solved. Due to the input constraints, the quasi-time-optimal control trajectories show bang-bang behavior. The performance of the numerically determined trajectories are demonstrated by means of measurement results on an experimental test bench.
\end{abstract}

Keywords: electromagnetic valve, fast-switching valve, soft landing, feedforward design, optimal control

\section{INTRODUCTION}

Fast-switching solenoid valves are used in various fields of applications. For instance in the manufacturing industry, fast sorting tasks are performed by means of short air pulses. In automation applications, pneumatic piston actuators are often controlled by means of pneumatic pulsewidth modulation and in the automotive industry, internal combustion engines are controlled by variable gas exchange timings. Common requirements of these applications are short switching times and minimal impact velocities of the plunger in order to eliminate acoustic noise, to avoid damage of mechanical components or at least to reduce mechanical wear. Different approaches reported in the literature address the soft landing problem, covering cycle adaptive controllers such as repetitive learning control (Tai et al., 2001), iterative learning control (Hoffmann et al., 2003) and extremum seeking (Peterson and Stefanopoulou, 2004). Feedback control strategies using state observers have been reported by Eyabi and Washington (2006); Peterson et al. (2006). Koch et al. (2002); Chung et al. (2007); Chladny and Koch (2008) enhanced these concepts by feedforward controllers, which are designed by exploiting the differential flatness properties of the underlying mathematical model. In order to minimize the transition time of the plunger from one valve seat to the other, the allowed input voltage range should be fully utilized. The flatness-based design methodology, however, does not allow to incorporate these constraints. One possibility of considering the input constraints in the flatness-based design is to assume a specific smooth trajectory shape, cf. Petit et al. (2001); Chung et al. (2007).

In this work, a feedforward controller for a fast-switching solenoid valve is designed by point-to-point quasi-timeoptimal control, which enables the incorporation of input constraints in a direct way. The work is structured as follows: In Section 2, a mathematical model of the consid- ered solenoid valve is developed, which is parametrized by nonlinear dynamic least-squares identification in Section 3. The optimal control problem is formulated in Section 4 and numerical results are shown in Section 5. Measurement results from an experimental test bench are given in Section 6 .

\section{MATHEMATICAL MODEL}

The mathematical model of the considered fast-switching valve can be separated into three subsystems: the model of the electromagnetic subsystem, the mechanical subsystem and the pneumatic subsystem. Since measurement results of the considered fast-switching valve confirm that the valve is pressure-balanced, the pressure forces acting on the plunger will be neglected. In addition, it will be assumed that the flow force is small in comparison to the magnetic force. Since no internal feedback from the pneumatic dynamics to the electromechanical subsystem is considered, the optimal control problem can be formulated with the pneumatic subsystem being neglected.

\subsection{Electromagnetic subsystem}

In Figure 1, the equivalent magnetic circuit of the fastswitching valve is given. It comprises the flux-dependent effective core reluctance $\mathcal{R}_{f c}\left(\Phi_{f c}\right)$, the effective reluctance $\mathcal{R}_{f p}$ of the plunger, the effective reluctance $\mathcal{R}_{g}(s)$ of the air gap between the core and the plunger, and the reluctance $\mathcal{R}_{l}$ which accounts for the leakage fluxes. The reluctances are modeled as

$$
\begin{aligned}
\mathcal{R}_{f c}\left(\Phi_{f c}\right) & =\frac{l_{f c}}{\mu_{0} \mu_{f c}\left(\Phi_{f c}\right) A_{f c}}, & \mathcal{R}_{f p} & =\frac{l_{f p}}{\mu_{0} \mu_{f p} A_{f p}}, \\
\mathcal{R}_{l} & =\frac{l_{l}}{\mu_{0} A_{l}}, & \mathcal{R}_{g}(s) & =\frac{2 s}{\mu_{0} A_{g}} .
\end{aligned}
$$

Here, $l_{f c}, l_{f p}$, and $l_{l}$ are the effective lengths of the core, the plunger, and the leakage flux lines, respectively. $A_{f c}$, 


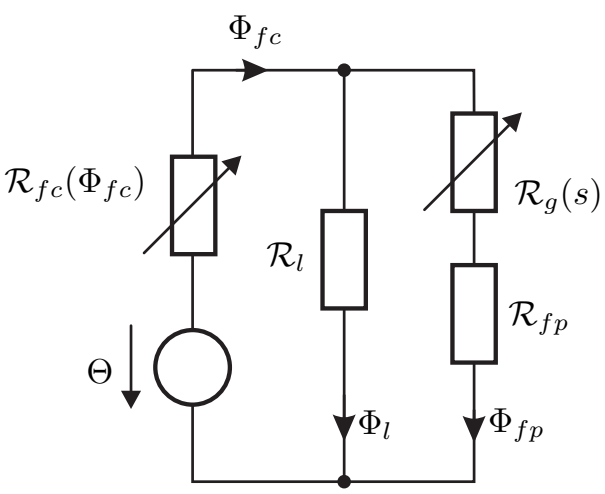

Fig. 1. Reluctance model of the fast-switching valve.

$A_{f p}$, and $A_{l}$ are the corresponding effective areas. The effective length of the air gap is $2 s$, since there are two air gaps between the core and the plunger, cf. Figure 2 . The corresponding area is denoted by $A_{g}$. Furthermore, $\mu_{0}$ denotes the permeability of air. The relative permeability $\mu_{f p}$ of the plunger is assumed to be constant. However, saturation of the core is phenomenologically modeled as

$$
\mu_{f c}\left(\Phi_{f c}\right)=\left(k_{1}\left(\frac{\left|\Phi_{f c}\right|}{A_{f c}}\right) \mathrm{e}^{k_{2} \frac{\left|\Phi_{f c}\right|}{A_{f c}}}+k_{3}\right)^{-1},
$$

with the constant parameters $k_{i}, i \in\{1,2,3\}$. From the magnetomotive force $\Theta=N i$, where $i$ is the current and $N$ is the number of windings, the flux $\Phi_{f_{c}}$ through the coil is given in the form

$$
\Phi_{f c}=\frac{\Theta}{\mathcal{R}} .
$$

Here, the equivalent reluctance $\mathcal{R}$ of the overall system reads as

$$
\mathcal{R}\left(s, \Phi_{f c}\right)=\mathcal{R}_{f c}\left(\Phi_{f c}\right)+\frac{\mathcal{R}_{l}\left(\mathcal{R}_{g}(s)+\mathcal{R}_{f p}\right)}{\mathcal{R}_{l}+\mathcal{R}_{g}(s)+\mathcal{R}_{f p}} .
$$

Based on the flux linkage $\psi=N \Phi_{f c}$ of the coil, Faraday's law yields

$$
\frac{\mathrm{d}}{\mathrm{d} t} \psi=-R i+v
$$

where $R$ is the electric resistance and $v$ is the voltage applied to the coil. The coil current $i$ can be expressed in terms of the flux linkage and the air gap as

$$
i=\frac{\mathcal{R}(s, \psi)}{N^{2}} \psi \text {. }
$$

From the magnetic energy

$$
\begin{aligned}
\mathcal{W}_{m} & =\int_{0}^{\psi} i(s, \bar{\psi}) \mathrm{d} \bar{\psi} \\
& =\int_{0}^{\psi} \frac{\mathcal{R}_{f c}(\bar{\psi})}{N^{2}} \bar{\psi} \mathrm{d} \bar{\psi}+\frac{1}{2 N^{2}} \frac{\mathcal{R}_{l}\left(\mathcal{R}_{g}(s)+\mathcal{R}_{f p}\right)}{\mathcal{R}_{l}+\mathcal{R}_{g}(s)+\mathcal{R}_{f p}} \psi^{2}
\end{aligned}
$$

the magnetic force $f_{m}$ (see Figure 2) can be deduced as

$$
\begin{aligned}
f_{m}(s, \psi) & =\frac{\partial \mathcal{W}_{m}}{\partial s} \\
& =\frac{1}{2 N^{2}} \frac{\mathcal{R}_{l}^{2}}{\left(\mathcal{R}_{l}+\mathcal{R}_{g}(s)+\mathcal{R}_{f p}\right)^{2}} \frac{\partial \mathcal{R}_{g}(s)}{\partial s} \psi^{2} .
\end{aligned}
$$

\subsection{Mechanical subsystem}

The overall mathematical model is completed by the mass balance of the plunger. Figure 2 shows a schematic

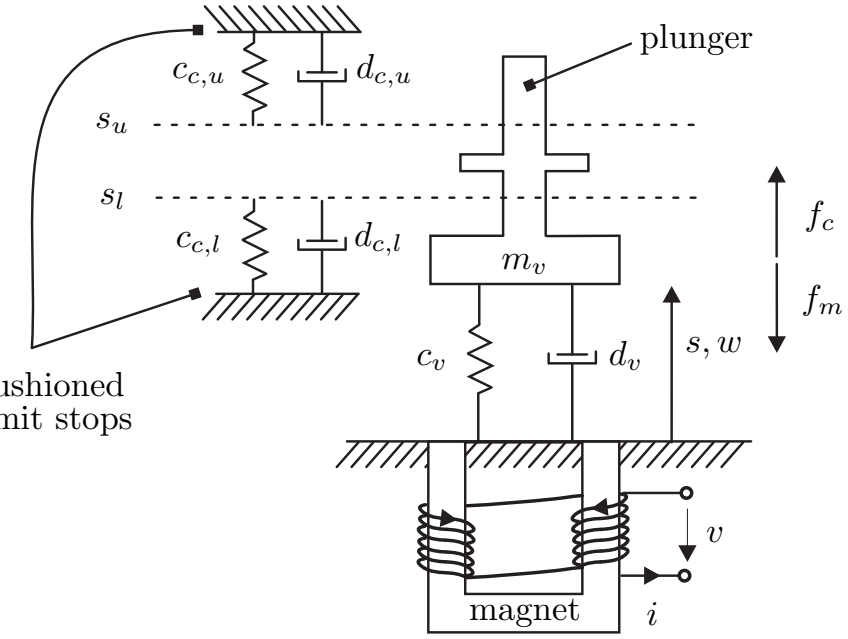

Fig. 2. Schematic diagram of the valve.

diagram of the fast-switching valve. Here, $w$ is the plunger velocity. The mass of the plunger is denoted by $m_{v}$, the stiffness of the load spring by $c_{v}$, and the viscous damping coefficient due to the friction of the housing and the sealing elements by $d_{v}$. The plunger is loaded by the magnetic force $f_{m}(s, \psi)$ and a contact force $f_{c}(s, w)$. The latter models the contact of the plunger with the lower and upper limit stops, which are reached at $s=s_{l}$ and $s=s_{u}$, respectively. That is, $f_{c}(s, w)=0 \forall s=\left[s_{l}, s_{u}\right]$. The mass balance of the plunger reads as

$$
\begin{aligned}
\frac{\mathrm{d}}{\mathrm{d} t} s & =w \\
\frac{\mathrm{d}}{\mathrm{d} t} w & =\frac{1}{m_{v}}\left(-f_{m}(s, \psi)+f_{c}(s, w)-c_{v}\left(s-l_{c_{0}}\right)-d_{v} w\right) .
\end{aligned}
$$

Herein, $c_{v} l_{c_{0}}$ denotes the preloading force resulting from the load spring. The limit stops of the plunger are cushioned by viscoelastic sealings, which can be described by means of a linear Kelvin-Voigt model

$$
f_{c}(s, w)= \begin{cases}-c_{c, u}\left(s-s_{u}\right)-d_{c, u} w & \text { for } s>s_{u} \\ -c_{c, l}\left(s-s_{l}\right)-d_{c, l} w & \text { for } s<s_{l} \\ 0 & \text { else. }\end{cases}
$$

Strictly speaking, the limit stop would entail a switched system with switching conditions at $s=s_{l}$ and $s=s_{u}$. The switching structure, however, may inordinately complicate the optimal control problem. Alternatively, the contact characteristics is smoothed a by tanh-function, i.e.,

$$
\begin{aligned}
f_{c}(s, w)= & \left(\alpha_{l}-\beta_{l} w\right) \frac{1}{2}\left(-\tanh \left(\eta_{l}\left(s-s_{l}\right)\right)+1\right) \\
& +\left(\alpha_{u}+\beta_{u} w\right) \frac{1}{2}\left(-\tanh \left(\eta_{u}\left(s-s_{u}\right)\right)-1\right) .
\end{aligned}
$$

The steepness of $f_{c}(s, w)$ can be adjusted by the parameters $\eta_{l}$ and $\eta_{u}$. Moreover, $\beta_{l}$ and $\beta_{u}$ denote the viscous damping coefficients. In order to approximately follow the linear spring characteristics at the contact points, the stipulation

$$
\left.\frac{\partial f_{c}}{\partial s}\right|_{s=s_{i}, w=0}=-c_{c, i} \approx-\frac{1}{2} \alpha_{i} \eta_{i}, \quad i \in\{l, u\}
$$

is made. The parameters $\alpha_{l}$ and $\alpha_{u}$ determine the maximal spring forces of the contact model. Figure 3 shows the 


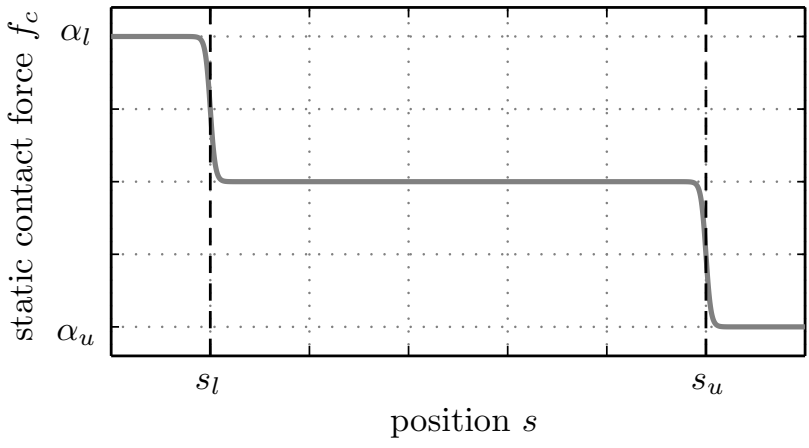

Fig. 3. Static contact force model.

smoothed contact characteristics (11) for the parameters $\beta_{u}=\beta_{l}=0$ and $\eta_{u}=\eta_{l}=10^{6} 1 / \mathrm{m}$.

\section{PARAMETER IDENTIFICATION AND MODEL VALIDATION}

Several parameters of the mathematical model cannot be directly determined from data sheets. Therefore, a parameter identification of the overall model is performed in order to fit the derived model to measurement results. First the electromagnetic subsystem is parametrized. For this, the reluctance (4) is reformulated in the structural equivalent form

$$
\mathcal{R}(s, \psi)=p_{1}|\psi| \mathrm{e}^{p_{2}|\psi|}+p_{3}+\frac{p_{4}\left(p_{5} s+p_{6}\right)}{p_{4}+p_{5} s+p_{6}},
$$

with the parameters

$$
\begin{array}{ll}
p_{1}=\frac{k_{1} l_{f c}}{\mu_{0} N A_{f c}^{2}}, & p_{2}=\frac{k_{2}}{N A_{f c}}, \quad p_{3}=\frac{k_{3} l_{f c}}{\mu_{0} A_{f c}}, \\
p_{4}=\mathcal{R}_{l}, & p_{5}=\frac{2}{\mu_{0} A_{g}}, \quad p_{6}=\mathcal{R}_{f p} .
\end{array}
$$

The unknown parameters $\boldsymbol{\theta} \in\left\{R, p_{1}, p_{2}, p_{3}, p_{4}, p_{5}, p_{6}\right\}$, with the electric resistance $R$ from (5), are found from the nonlinear dynamic least-squares identification task

$$
\begin{array}{rlrl}
\min _{\boldsymbol{\theta}} & \frac{1}{T} \int_{0}^{T}\left(i-i_{m}\right)^{2} \mathrm{~d} t \\
\text { s.t. } & \frac{\mathrm{d}}{\mathrm{d} t} \psi & =v-R i, \quad \psi(0)=\psi_{0} \\
i & =\frac{\mathcal{R}\left(s_{m}, \psi\right)}{N^{2}} \psi .
\end{array}
$$

Here, $i_{m}$ and $s_{m}$ are the measurements of the current and the plunger position, respectively. The measurements were carried out during the time interval $t \in(0, T)$ for different steps in the input voltage $v$.

Figure 4 shows results of the parametrized model for two different voltage steps $v$. The results confirm that the reluctance model (4) is capable of approximating the real electromagnetic behavior of the valve in an excellent way. The effect of saturation is negligible for small current values, cf. Figure 4(a). However, for large currents, Figure 4(b) clearly demonstrates the necessity of modeling the nonlinear saturation phenomena. In addition, the inductance $L=\mathcal{R}(s, \psi) / N^{2}$ is shown in Figure 4 .

The magnetic force $f_{m}$ is calculated from the identified reluctance model according to (8). Due to temperature dependency and the presence of hysteresis of the viscoelastic material, the proposed force model (11) is only an approximation of the real viscoelastic behavior. Thus, the identification of the mechanical parameters from the mass balance (9) by applying again nonlinear dynamic leastsquares identification fails. In fact, the spring constant $c_{v}$ as well as the preloading force $c_{v} l_{c_{0}}$ were obtained from measurements in stationary conditions. The parameters of the contact model and the viscous damping coefficient $d_{v}$ were adjusted in order to approximately reproduce the contact behavior with the sealing. Based on the developed model, the optimal control problem is formulated in the next section.

\section{OPTIMAL CONTROL PROBLEM}

The mathematical model (5) and (9) with the state vector $\boldsymbol{x}=[s, w, \psi]^{T} \in \mathbb{R}^{3}$ with the constrained affine input $u=v \in \mathbb{R}$ can be written in the form

$$
\frac{\mathrm{d}}{\mathrm{d} t} \boldsymbol{x}=\boldsymbol{f}(\boldsymbol{x})+\boldsymbol{b} u, \boldsymbol{x}(0)=\boldsymbol{x}_{0},
$$

with the initial condition $\boldsymbol{x}_{0} \in \mathbb{R}^{3}$. The control objective is to find an optimal control $u^{*} \in \mathcal{U}=\left[u^{-}, u^{+}\right]$that guarantees a minimal transition time $T_{f}$ for a set-point change

with

$$
\left(u_{0}, \boldsymbol{x}_{0}\right) \rightarrow\left(u_{f}, \boldsymbol{x}_{f}\right), \quad t \in\left(0, T_{f}\right)
$$

$$
\begin{aligned}
u(0)=u_{0}, & \mathbf{0}=\boldsymbol{f}\left(\boldsymbol{x}_{0}\right)+\boldsymbol{b} u_{0}, \\
u\left(T_{f}\right)=u_{f}, & \mathbf{0}=\boldsymbol{f}\left(\boldsymbol{x}_{f}\right)+\boldsymbol{b} u_{f}
\end{aligned}
$$

and the terminal condition $\boldsymbol{x}\left(T_{f}\right)=\boldsymbol{x}_{f} \in \mathbb{R}^{3}$. Therefore, the input-constrained point-to-point optimal control problem

$$
\begin{array}{ll}
\min _{u \in \mathcal{U}} & J(u)=\varphi\left(T_{f}\right)+\int_{0}^{T_{f}} l(u) \mathrm{d} t \\
\text { s.t. } & \frac{\mathrm{d}}{\mathrm{d} t} \boldsymbol{x}=\boldsymbol{f}(\boldsymbol{x})+\boldsymbol{b} u, \boldsymbol{x}(0)=\boldsymbol{x}_{0}, \boldsymbol{x}\left(T_{f}\right)=\boldsymbol{x}_{f}, \\
& u \in \mathcal{U}=\left[u^{-}, u^{+}\right]
\end{array}
$$

has to be solved. The summand $\varphi\left(T_{f}\right)=T_{f}$ of the cost functional $J(u)$ represents the time optimality, whereas the integral term $l(u)=r u^{2} / 2$, with $r>0$, serves as a regularization in order to avoid singular arcs in the quasi-time-optimal control problem. Introducing the Hamiltonian (Bryson and Ho, 1975)

$$
\mathcal{H}(\boldsymbol{x}, u, \boldsymbol{\lambda})=l(u)+\boldsymbol{\lambda}^{T}(\boldsymbol{f}(\boldsymbol{x})+\boldsymbol{b} u),
$$

with the adjoint states $\boldsymbol{\lambda} \in \mathbb{R}^{3}$, and applying a time transformation $t=T_{f} \tau$ that maps the time interval $t \in\left(0, T_{f}\right)$ onto $\tau \in(0,1)$, the optimal control problem can be reformulated by means of Pontryagin's maximum principle (Athans and Falb, 1966) in form of a two-point boundary value problem, i.e.

$$
\begin{aligned}
\frac{\mathrm{d}}{\mathrm{d} \tau} \boldsymbol{x}^{*} & =T_{f}\left(\boldsymbol{f}\left(\boldsymbol{x}^{*}\right)+\boldsymbol{b} u^{*}\right) \\
\frac{\mathrm{d}}{\mathrm{d} \tau} \boldsymbol{\lambda}^{*} & =-\left.T_{f}\left(\frac{\partial}{\partial \boldsymbol{x}} \boldsymbol{f}\right)^{T}(\boldsymbol{x})\right|_{\boldsymbol{x}=\boldsymbol{x}^{*}} \boldsymbol{\lambda}^{*} \\
u^{*} & =\underset{u \in \mathcal{U}}{\arg \min } \mathcal{H}\left(\boldsymbol{x}^{*}, u, \boldsymbol{\lambda}^{*}\right)
\end{aligned}
$$

with boundary conditions

$$
\boldsymbol{x}^{*}(0)=\boldsymbol{x}_{0} \quad \text { and } \quad \boldsymbol{x}^{*}(1)=\boldsymbol{x}_{f}
$$

and the transversality condition

$$
\left.\mathcal{H}\left(\boldsymbol{x}^{*}, u^{*}, \boldsymbol{\lambda}^{*}\right)\right|_{\tau=1}=-1
$$




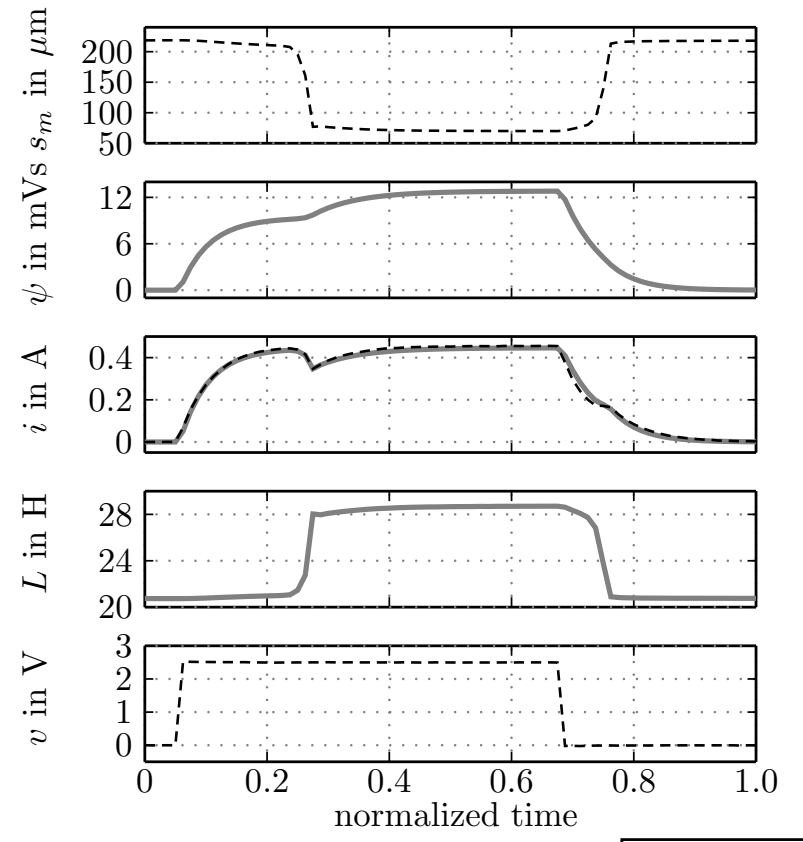

(a) Set-point change of $v=2.5 \mathrm{~V} \cdot\left[\begin{array}{cc}\stackrel{\text { Simulation }}{---} & \text { Measurement } \\ - & \end{array}\right.$
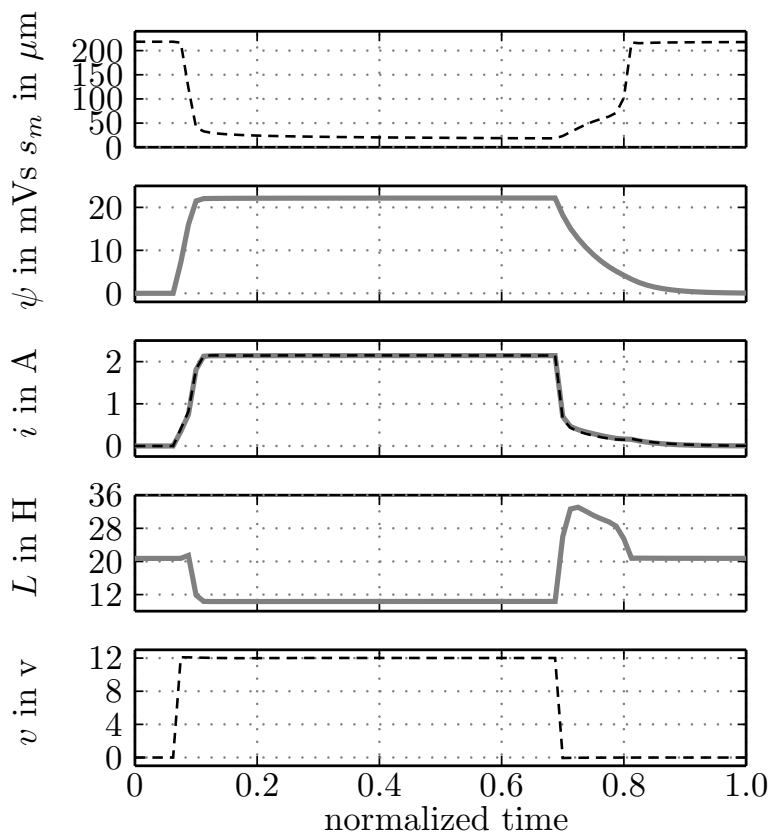

(b) Set-point change of $v=12 \mathrm{~V}$.

Fig. 4. Identification results for different voltage set-point changes. Measured air gap $s_{m}$, simulated flux linkage $\psi$, measured and simulated current $i_{m}$ and $i$, and simulated inductance $L$.
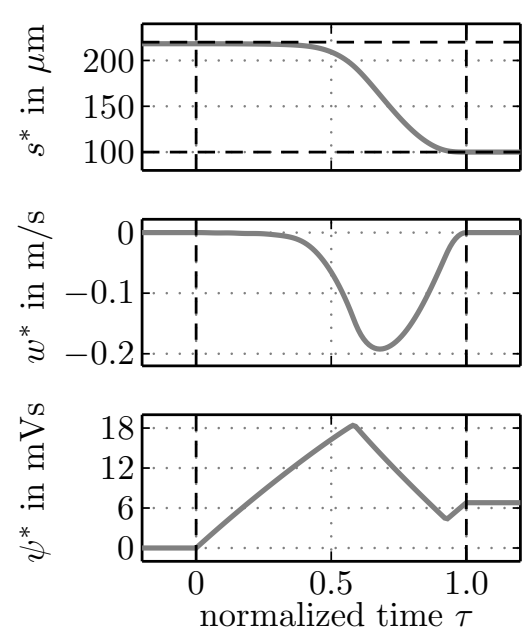

(a) Optimal state trajectories.
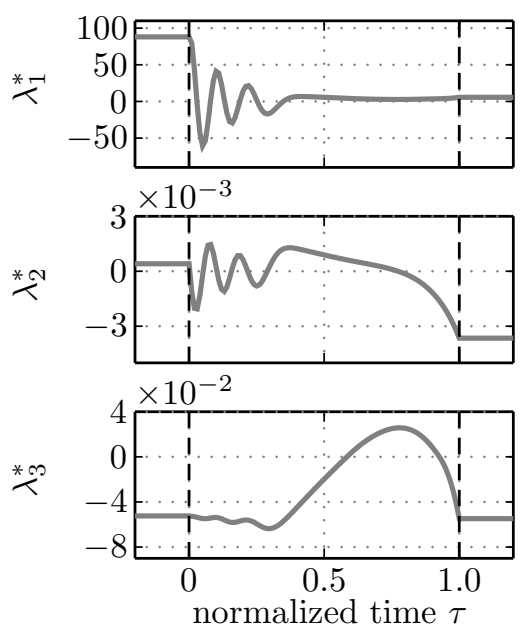

(b) Optimal adjoint state trajectories.
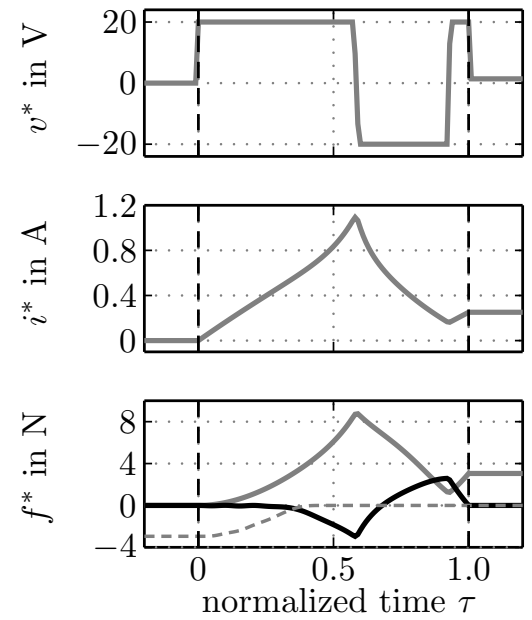

(c) Optimal voltage $v^{*}$, current $i^{*}$, magnetic force $f_{m}^{*}(-)$, contact force $f_{c}^{*}(----)$ and sum force $f_{\text {sum }}^{*}(\longrightarrow)$.

Fig. 5. Numerical results of the time optimal control problem for the opening motion $\left(s_{u} \rightarrow s_{l}\right)$.

resulting from the free end time $T_{f}$. The superscript * refers to optimal variables. Owing to the input affine system representation (16), the minimization problem (21c) can be explicitly solved resulting in an optimal control function

$$
u^{*}=\xi(\boldsymbol{\lambda})= \begin{cases}u^{-} & \text {for } \quad u^{0} \leq u^{-} \\ u^{0} & \text { for } \quad u^{0} \in\left(u^{-}, u^{+}\right) \\ u^{+} & \text {for } \quad u^{0} \geq u^{+}\end{cases}
$$

with

$$
u^{0}=-\frac{1}{r}\left(\boldsymbol{\lambda}^{*}\right)^{T} \boldsymbol{b}
$$

Note that $\boldsymbol{\lambda}^{T} \boldsymbol{b}=\lambda_{3}$. In the limit case for $r \rightarrow 0$ each time $\lambda_{3}$ crosses zero, $u^{*}$ switches between the limits $u^{-}$ and $u^{+}$. Then, the solution of the optimal control problem (19) is a bang-bang control, except for $\lambda_{3}=0$. Also note that for $r=0$ the optimal control problem (19) is singular (Bryson, 1999) since $u$ can neither be derived from the minimization problem (21c) nor from the related first-order necessary condition $\partial \mathcal{H} / \partial u=0$. Then, the optimal control input may be deduced from some total time derivatives of $\partial \mathcal{H} / \partial u=0$, cf. Kelley et al. (1967). 

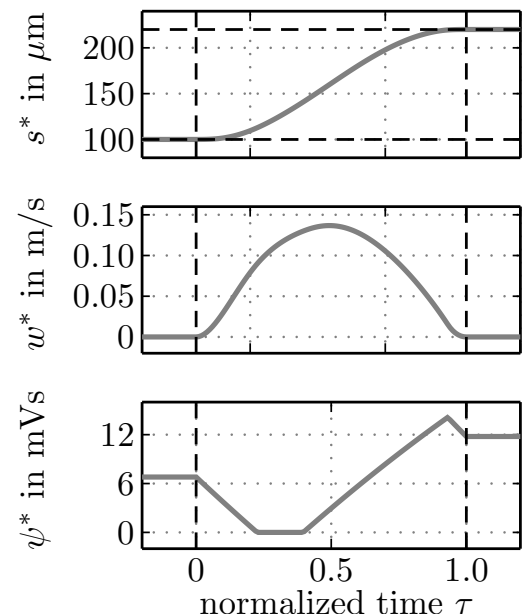

(a) Optimal state trajectories.
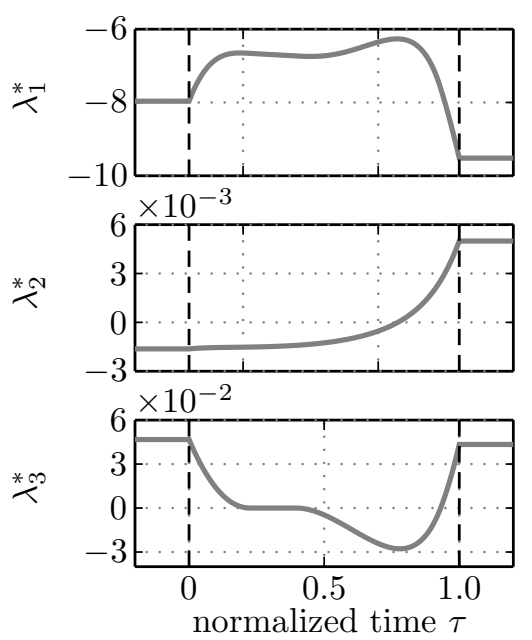

(b) Optimal adjoint state trajectories.
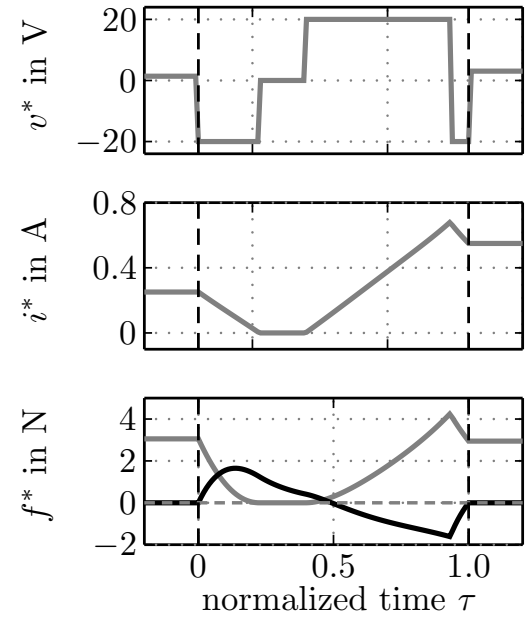

(c) Optimal voltage $v^{*}$, current $i^{*}$, magnetic force $f_{m}^{*}(-)$, contact force $f_{c}^{*}(----)$ and sum force $f_{\text {sum }}^{*}(\longrightarrow)$.

Fig. 6. Numerical results of the time optimal control problem for the closing motion $\left(s_{l} \rightarrow s_{u}\right)$.

\section{NUMERICAL RESULTS OF THE OPTIMAL CONTROL PROBLEM}

Numerical solutions of the two-point boundary value problem (21)-(23) can be obtained using the MATLAB function bvp4c(), cf. Shampine et al. (2003). Figures 5 and 6 show numerical results of the time-optimal point-to-point transition for the opening

$$
\left(u_{0}=0, \boldsymbol{x}_{0}=\left[s_{u}, 0,0\right]^{T}\right) \rightarrow\left(u_{f}=v_{l}, \boldsymbol{x}_{f}=\left[s_{l}, 0, \psi_{l}\right]^{T}\right)
$$

and for the closing

$\left(u_{0}=v_{l}, \boldsymbol{x}_{0}=\left[s_{l}, 0, \psi_{l}\right]^{T}\right) \rightarrow\left(u_{f}=v_{u}, \boldsymbol{x}_{f}=\left[s_{u}, 0, \psi_{u}\right]^{T}\right)$ of the fast-switching valve within the normalized transition time $\tau \in(0,1)$. Here, $v_{l}$ and $\psi_{l}$ denote the resulting set-point voltage and flux linkage, respectively, cf. (18), at the upper limit, and $v_{u}$ and $\psi_{u}$ the set-point voltage and flux linkage at the lower limit. Note that for all numerical solutions outside the vertical dashed lines the initial and final values are held constant for illustration purposes only. The optimal state trajectories for opening the valve are given in Figure 5(a). Figure 5(c) shows the corresponding optimal voltage $v^{*}$, which is nearly bangbang because $r$ in the penalty term is chosen very small $r=10^{-8} 1 / \mathrm{V}^{2}$. Moreover, Figure $5(\mathrm{c})$ contains the optimal current $i^{*}$, the magnetic force $f_{m}^{*}$, the contact force $f_{c}^{*}$ and the sum of the forces $f_{\text {sum }}^{*}$ acting on the plunger. The optimal adjoint states are shown in Figure 5(b). They exhibit oscillations at the beginning of the considered time interval. Although the incorporation of the contact model (11) results in a locally numerical stiff model, the oscillation is not a numerical artifact but inherent to the model. Numerical results for different contact stiffnesses $c_{c_{i}}, i \in\{u, l\}$, of the contact model confirm this statement. Figure 6 shows analogous numerical results for closing the valve with $r=10^{-8} 1 / \mathrm{V}^{2}$. It is worth noting that in this case the solution of the optimal control problem is not purely bang-bang. Whenever $\lambda_{3}^{*}$ vanishes (approximately for $\tau \in[0.4,0.6]), u^{*}$ does so as well. This happens if $f_{m}^{*}$ vanishes, implying that only the spring force accelerates the plunger. For confirming the numerical results of the two-point boundary value problem, the optimal control problem was additionally solved by full discretization with SNOPT, see Gill et al. (2006), resulting in the same optimal control trajectories.

\section{IMPLEMENTATION AND EXPERIMENTAL RESULTS}

The obtained numerical results were verified on an experimental test bench. The power electronics used for driving the magnetic valve allows the accurate measurement of the current and the voltage. The position and velocity of the plunger were obtained from a laser vibrometer (POLYTEC) and the measuring and control system DSPACE 1005 was utilized for data processing.

The experimental results revealed small model inaccuracies, which may be attributed to temperature dependency of the coil resistance and of the contact behavior of the limit stops and to stick-slip effects. However, by slightly moving the switching-points of the input voltage, it is possible to open and close the valve in minimal time and with almost zero velocity at the limit positions. Figure 7 shows the measurement results for the opening and the closing of the magnetic valve. For the opening scenario in Figure $7(\mathrm{a})$, in contrast to the numerical results, the normalized opening time of $\tau_{m}=0.87 \tau$ was needed. It is evident, that by applying the structural equivalent input trajectory it is possible to minimize the impact velocity at the seals. The same applies to the valve closing in Figure 7(b). A normalized time ratio of $\tau_{m}=0.84 \tau$ was needed for the set-point change within minimal time.

\section{CONCLUSION AND FUTURE WORK}

In this work, time-optimal feedforward trajectories for fast-switching valves are presented. Nonlinear dynamic least-squares identification was performed to parametrize 

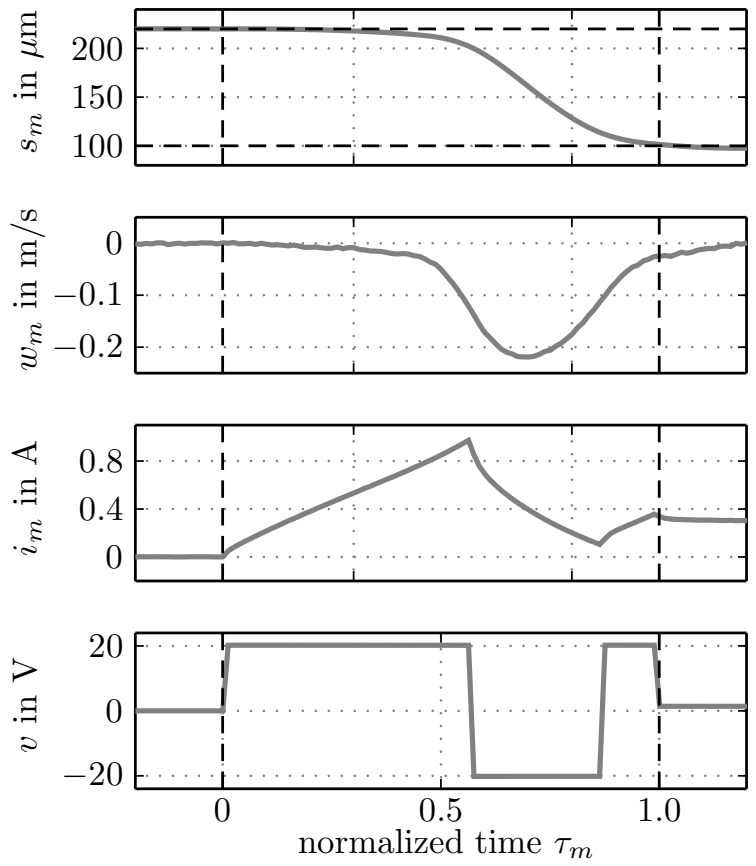

(a) Opening motion $\left(s_{u} \rightarrow s_{l}\right)$.
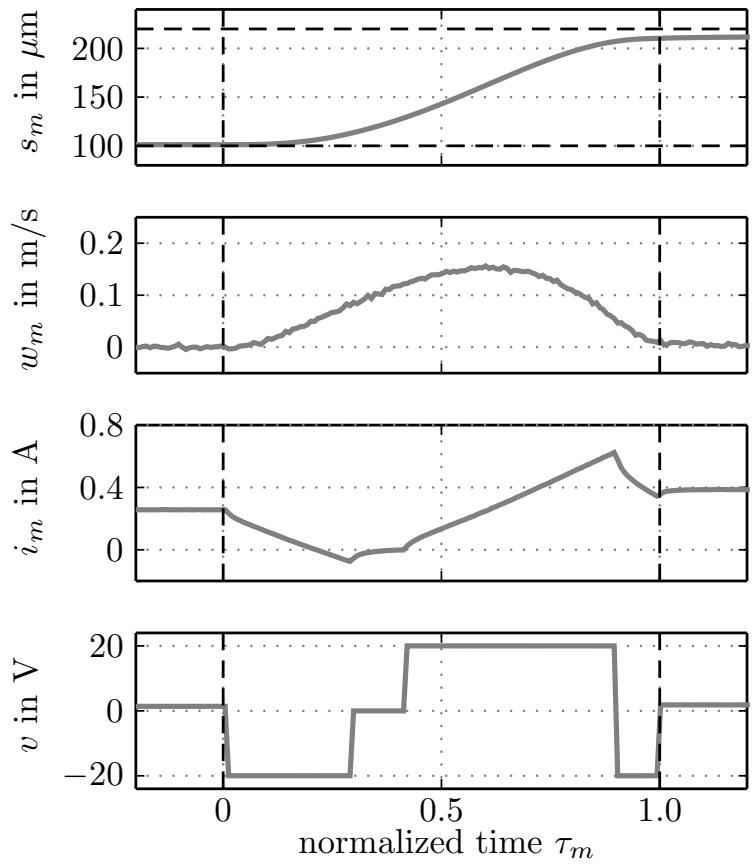

(b) Closing motion $\left(s_{l} \rightarrow s_{u}\right)$.

Fig. 7. Measurement results from an experimental test bench.

the developed mathematical model. The point-to-point quasi-time-optimal control problem was reformulated by means of Pontryagin's maximum principle and numerically solved by a direct approach. In the last part, the applicability of the time-optimal feedforward trajectories is demonstrated by means of measurement results. Future work addresses the cycle-based adaption of the time-optimal feedforward trajectory in order to account for time-varying parameters and model mismatches.

\section{ACKNOWLEDGEMENTS}

We thank Prof. Dr.-Ing Knut Graichen for his helpful discussion on formulating and solving the optimal control problem.

\section{REFERENCES}

Athans, M. and Falb, P. (1966). Optimal Control: An Introduction to the Theory and Its Applications. McGrawHill, New York.

Bryson, A. (1999). Dynamic optimization. AddisonWesley.

Bryson, A. and Ho, Y. (1975). Applied optimal control. John Wiley \& Sons, New York.

Chladny, R.R. and Koch, C.R. (2008). Flatness-based tracking of an electromechanical variable valve timing actuator with disturbance observer feedforward compensation. IEEE Transactions on Control System Technology, 16(4), 652-663.

Chung, S.K., Koch, C.R., and Lynch, A.F. (2007). Flatness-based feedback control of an automotive solenoid valve. IEEE Transactions on Control System Technology, 15(2), 394-401.

Eyabi, P. and Washington, G. (2006). Modeling and sensorless control of an electromagnetic valve actuator. Mechatronics, 16, 159-175.
Gill, P.E., Murray, W., and Sauders, M.A. (2006). User's Guide for SNOPT Version 7: Software for LargeScale Nonlinear Programming. URL http://www. sbsi-sol-optimize.com.

Hoffmann, W., Peterson, K., and Stefanopoulou, A.G. (2003). Iterative learning control for soft landing of electromechanical valve actuator in camless engines. IEEE Transactions on Control System Technology, 11(2), 174184.

Kelley, H., Kopp, R., and Moyer, H. (1967). Singular extremals, chapter 3, 63-101. Academic Press.

Koch, C.R., Lynch, A., and Chladny, R. (2002). Modeling and control of solenoid valves for internal combustion engines. In Proceedings of the IFAC Symposium on Mechatronic Systems, 197-201. California, USA.

Peterson, K.S., Grizzle, J.W., and Stefanopoulou, A.G. (2006). Nonlinear control for magnetic levitation of automotive engine valves. IEEE Transactions on Control System Technology, 14(2), 346-354.

Peterson, K.S. and Stefanopoulou, A.G. (2004). Extremum seeking control for soft landing of an electromechanical valve actuator. Automatica, 40, 1063-1069.

Petit, N., Milam, M.B., and Murray, R.M. (2001). Inversion based constrained trajectory optimization. In Proceedings of the 5th IFAC Symposium on Nonlinear Control Systems. St. Petersburg, Russia.

Shampine, L., Gladwell, I., and Thompson, S. (2003). Solving ODEs with MATLAB. Cambridge University Press.

Tai, C., Stubbs, A., and Tsao, T. (2001). Control of an electromechanical actuator for camless engines. In Proceedings of the American Control Conference, 31133118. 